\title{
Kinetics of the Carbon-Oxygen Reaction in Liquid Iron*
}

\author{
By Kichizo Niwa**, Mitsuo Shimoji**, \\ Tamiya Kishida*** and Yukiyoshi Itoh****
}

\begin{abstract}
Synopsis
Reaction mechanisms of $\mathrm{CO}$ evolution in liquid iron-carbon alloys were investigated and the rates of carbon oxidation were measured by blowing oxygen into liquid iron. Since an estimated rate of the homogeneous reaction is extremely rapid at $2000^{\circ} \mathrm{K}$, the rate of $\mathrm{C}-\mathrm{O}$ reaction can be determined by reactant transports from the uniform bulk to the equilibrium area. Mathematical expressions are given to nucleation, growth, and motion of $\mathrm{CO}$ bubbles in liquid iron, and reaction rates in open hearths. Measured rates of $\mathrm{C}+\frac{1}{2} \mathrm{O}_{2}(\mathrm{~g})$ reaction are interpreted on the basis of the present formulation.
\end{abstract}

\section{Homogeneous Chemical Reaction}

The theory of absolute reaction rates developed by Eyring ${ }^{1)}$ provides an estimated rate of a chemical reaction. If the rate of the carbon-oxygen reaction in liquid iron were controlled by the process of "homogeneous one", the rate of this reaction would be approximately

$$
-\frac{d[\mathrm{C}]}{d t}=\frac{R T}{N_{o} h} e \times p\left(-\frac{\Delta H^{\ddagger}}{R T}\right) \frac{7}{1600}[\mathrm{C}][\mathrm{O}]
$$

where $[\mathrm{C}]$ and $[\mathrm{O}]$ are respectively weight per cent of carbon and that of oxygen in liquid iron, $R$ gas constant, $T$ absolute temperature, $N_{0}$ Avogadro number, $h$ Planck's constant, and $\Delta H^{\neq}$ activation energy. Putting $T=2000^{\circ} \mathrm{K}$ and $\Delta H^{\ddagger}=$ 28 Kcal.,2) estimated $-d[\mathrm{C}] / d t \cdot[\mathrm{C}][\mathrm{O}]$ is found to be roughly $10^{7}$ per cent per hour. This result is too large compared to the observed value. The same conclusion has been obtained in Darken's calculation $^{3)}$ for heterogeneous chemical reaction on the surface of carbon monoxide bubbles. It is to be noted that the rate of homogeneous reaction is not dependent on the bath size of the liquid metal (or the contents of the charged metal) as shown in equation (1.1). However, in the case of the heterogeneous reaction the bath size has an inverse effect on the rate. In other words, a large bath size results in a low reaction rate and vice versa.

\section{Diffusion Film Theory}

The rate of $\mathrm{CO}$ formation may thus be rapid in terms of chemical reaction, whether it is homogeneous or heterogeneous. Now the most reasonable process for determining $\mathrm{CO}$ formation would be a transfer of the dissolved carbon (C) and oxygen $(\mathrm{O})$ to the existing surface of $\mathrm{CO}$ bubbles.
Generally, migration of the materials in liquids is due to diffusion, stirring, and convection, etc., where a uniform concentration rapidly prevails in the liquid state as compared to the solid state. However, there is occasionally a concentration gradient in the liquid near the interface between heterogeneous phases as shown in the solution process of a solid or gas into a liquid. This may be owing to viscous effects of the liquid near the boundary surface. The analogous effect is found in fluid dynamics. For example, in a fluid of Raynolds' number $\mathrm{R}^{*}$, a solid material of dimension $r$ has the thickness of the boundary layer, influenced by the viscosity of the liquid, $\Delta l$, given by

$$
\Delta l \cong r / \sqrt{ } R^{*}
$$

Reynolds' number $R^{*}$ in the case of CO gas bubbles in liquid iron is evaluated as follows:

$$
R^{*}=\rho v r / \eta
$$

where $\rho$ is the density of the liquid iron, $v$ the velocity of the bubble, $r$ the radius, $\eta$ the viscosity coefficient of liquid iron. As far as $v \cong 1 \mathrm{~cm} / \mathrm{sec}$, which will be described later, and $r \cong 1 \mathrm{~cm}, R$ in (2.2) is roughly $10^{3}$, since $\rho=7$ and $\eta \cong 10^{-2}$ poise. Consequently, the thickness of the surface layer of a gas sphere with a radius of $1 \mathrm{~cm}$ will be roughly $0.03 \mathrm{~cm}$. Similar consideration will be possible also in the case of liquid metal-slag interface or-air flat interface. The latter case has been observed, by Shvartzman, Samarin, and Temkin in their experiments on the rate of carbon oxidation ${ }^{4}$. In this case the thickness is $0.01 \mathrm{~cm}$. The order of magnitude of this thickness is concordant with the theoretical value. Thus, the viscous effect of liquid iron is regarded as an important cause for the concentration gradient of $\underline{C}$ and $O$ near the gas bubbles and slag or solid surfaces. Then, this concentration gradient in the narrow region near the surface of the heterogeneous phase $\left(1^{-2}\right.$ or $10^{-3} \mathrm{~cm}$ ) may give the respective rate determining steps of many reaction in liquid iron.

The stationary flows of the dissolved carbon and oxygen through the boundary layer are given by 35$)$ (5)

$$
\begin{gathered}
J_{c}=D_{c} \begin{array}{cc}
N_{o} \rho\left([\mathrm{C}]-[\mathrm{C}]_{\Omega}\right) & \text { atoms } \\
1200 \Delta l & \mathrm{~cm}^{2} \mathrm{sec}
\end{array} \\
J_{o}=D_{o} \begin{array}{c}
N_{o} \rho\left([\mathrm{O}]-[\mathrm{O}]_{\Omega}\right) \\
1600 \Delta l
\end{array} \\
\begin{array}{cc}
\text { atoms } \\
\mathrm{cm}^{2} \mathrm{sec}
\end{array}
\end{gathered}
$$

In these equations $D_{c}$ and $D_{o}$ are the diffusion

* Lecture delivered before the 55th Grand Lecture Meeting of the Iron \& Steel Institute of Japan, on April 2, 1958, in Tokyo. Japanese text was printed in "Tetsu-to-Hagané" (Journal, Iron \& Steel Institute, Japan) Vol. 46, No. 1, pp. 19-24, Vol. 47, No. 2, pp. 111-115.

** Department of Chemistry, Faculty of Science, Hokkaido University, Japan.

*** Yasuki Works, Hitachi Metal Industries, Ltd.

**** Muroran Works, Fuji Iron \& Steel Co., Ltd. 
coefficients for $\mathrm{C}$ and $\mathrm{O}, \Delta l$ represents boundary layer thickness, $[\mathrm{C}]_{\Omega}$ and $[\mathrm{O}]_{\Omega}$ respectively are weight per cents of $\mathrm{C}$ and $\mathrm{O}$ on the surface. Of course, an important relation

$$
J_{c}=J_{o}=J
$$

must hold always, in order to realize the stoichiometric chemical reaction and to reach chemical equilibrium on the gas-metal interface. This relation has also been adopted in their kinetic study of C-O evolution by Parlee, Seagle and Schuh$\left.\operatorname{mann}{ }^{\pi}\right)$

\section{Nucleation of CO Bubbles}

The problem of $\mathrm{CO}$ bubble formation in liquid iron belongs to a sort of phase transformation. Then this process can be treated by means of some method like Volmer-Becker-Döring's theory ${ }^{8)}{ }^{9}$.

In the case of carbon oxidation the free energy change (per molecule)

$$
\Delta G_{c o}=\frac{R T}{N_{o}} l_{n} \quad P_{c o}
$$

must have a negative value. In equation (3.1), $K_{c o}$ is the equilibrium constant for the reaction

$$
\underline{\mathrm{C}}+\underline{\mathrm{O}} \rightarrow \mathrm{CO}(g)
$$

and $P_{c o}$ is the partial pressure of $\mathrm{CO}$ in the bulk gas phase. However, equation (3.1) needs a correction due to surface effect if the size of the gas bubble follows progressive processes:

$$
\begin{aligned}
& \underline{\mathrm{C}}+\underline{\mathrm{O}}+\rightarrow \mathrm{CO} \\
& \mathrm{CO}+\underline{\mathrm{C}}+\underline{\mathrm{O}} \rightarrow(\mathrm{CO})_{2} \\
& \cdots \ldots \ldots \ldots \ldots \\
& (\mathrm{CO})_{n-1}+\underline{\mathrm{C}}+\underline{\mathrm{O}} \rightarrow(\mathrm{CO})_{n}
\end{aligned}
$$

where $n$ means the number of $\mathrm{CO}$ molecules in the bubble. Hence, the free energy for forming a bubble containing $n$ molecules from the dissolved carbon and oxygen in liquid iron is given by

$$
\Delta G_{n}=n \Delta G_{c o}+\sigma S_{n}
$$

where $\sigma$ is the surface energy of liquid iron per unit area, and $S_{n}$ is the surface area of the bubble. The first term in equation (3.4) has a negative value, while the second is positive. Then, $\Delta G_{n}$ must pass through a maximum, at $n=n^{*}$. In other words, the wroth of sub-microscopic bubbles of $\mathrm{CO}$ should be permitted to begin after the number of molecules overcomes this critical number $n$ *. The critical size of the present gas bubble, $r *$, may be evaluated approximately as follows. The relation between the pressure of the bubble and the number of the molecules in the bubble may be described by

$$
\left(P+\frac{2 \sigma}{r}\right) \frac{4}{3} \pi r^{3}=\frac{n R T}{N_{o}}
$$

where $r$ is the radius of the gas bubble, and $P$ is the pressure of the atmosphere plus the ferrostatic pressure. Putting $S_{n}=4 \pi \mathrm{r}^{2}$, one obtains

$$
r^{*}=\left(-\frac{2 R T}{N_{o} \Delta G_{c o}}-\frac{4}{3}\right) \frac{\sigma}{P}
$$

from equation (3.5) and

$$
\left(\partial \Delta G_{n} / \partial r\right)_{r=r^{*}}=0
$$

Using $[\mathrm{O}]_{c e}$, which is defined by

$$
[\mathrm{O}]_{c e}=\frac{K_{c o}}{[\mathrm{C}]}
$$

one has

$$
r^{*}=\left[\frac{2}{l_{n}\left([\mathrm{O}] /[\mathrm{O}]_{c e}\right)}-\frac{4}{3}\right] \frac{\sigma}{P}
$$

The free energy change of critical size of the gas bubble is given by

$$
\Delta G^{*}=4 \pi \sigma r^{* 2}\left[1-\frac{\frac{4 \sigma}{r^{*}}+2 P}{\frac{4 \sigma}{r^{*}}+3 P}\right]
$$

Inserting, $\quad r^{*} \cong 10^{-3} \mathrm{~cm}$ and $\sigma \cong 1500$ dyne $/ \mathrm{cm}$ into (3.10), one will find that $\Delta G^{*}$ is extremely large. Consequently, the actual origin of bubble formation should exist in another place, where easier processes prevail in terms of energy. These places may be contact surfaces between porous solids and liquids, e.g., the surfaces of furnace bottoms, brick walls, limestone, ore materials and scrap metals, etc.

\section{The Motion of CO Bubbles}

As has been discussed by many investigators, the favorable place of origin for bubble formation would be in the solid-liquid interfaces ${ }^{(6)}$. The holes of the solid surface may not be entirely filled under the influence of high surface tension of liquid metals, thus creating small gas pockets. These tiny gaseous spaces on the interfaces facilitate continuous bubble formation. If the holes or cavities are assumed to be a cylinder with radius $d$, favorable condition for bubble nucleation is that $d>r^{*}$. This is because of the impossibility of formation of smaller bubbles due to the existence of a large amount of Gibbs free energy change for nucleation of gas bubbles with radius $r^{*}$ as shown in the preceding section.

The motion of gas bubbles in liquid iron may be described by the Stokes-Navier equation. If the viscosity of liquid iron denoted by $\eta$ were large enough (i.e. small Reynolds number), the famous Stokes law

$$
F_{R S}=6 \pi \eta r v
$$

could be used to express the frictional force of a sphere having radius $r$ and velocity $v$ in liquids. The buoyancy of the gas bubble must be balanced by this frictional force. Thus, 


$$
v=\frac{2}{9} \frac{g}{\eta} \rho r^{2}
$$

Using $\rho \cong 7, \eta \cong 2 \times 10^{-2}$ poise, one obtains $v \cong 700$ $\mathrm{m} / \mathrm{second}$ for radius of bubble, $1 \mathrm{~cm}$. This figure leads to $R^{*} \cong 10^{5}$ in equation (2.2). Therefore, Newton's equation should be used in place of equation (4.1). That is roughly

$$
F=\pi r^{2} \rho v^{2}
$$

Thus, one obtains

$$
v=\left(\frac{4}{3} r g\right)^{\frac{1}{2}}
$$

Then, the velocity of gas bubbles with a radius of $1 \mathrm{~cm}$ is less than $40 \mathrm{~cm} / \mathrm{sec}$. This figure in liquid irons is concordant with the above discussion in section 2, in which the large Reynolds number results in the formation of the boundary interface layer.

As stressed by Darken") and Larsen ${ }^{6)}$, the size of the gas bubble in the open hearth is supposed to have a dimension of about $1 \mathrm{~cm}$. Consequently, the present discussion may be a reasonable explanation for the phenomena concerning the gas bubbles in liquid iron.

\section{Rate of Carbon-Oxygen Reaction in the Open Hearth}

This problem has been extensively discussed by Darken3)5). Here, a modified consideration is presented from a similar point of view. As shown in equation (2.4), the flow of carbon and oxygen from a bulk liquid to the surface being in chemical equilibrium must have the same number of respective atoms. Thus, one obtains

$$
\begin{aligned}
& -\frac{d[\mathrm{C}]}{d t}=\frac{3}{4} D_{o} \frac{[\mathrm{O}]-[\mathrm{O}] \Omega_{e}}{l \Delta l} \frac{S_{g}}{S_{m}} \\
& -\frac{d[\mathrm{C}]}{d t}=D_{c}[\mathrm{C}]-[\mathrm{C}] \Omega_{e} \frac{S_{g}}{l \Delta l}
\end{aligned}
$$

where $l$ is the depth of the liquid metals, $S_{g}$ is the surface area between gas and metal, and $\mathrm{S}_{m}$ is that between slag and metal. $[\mathrm{O}]_{\Omega_{e}}$ and $[\mathrm{C}]_{\Omega_{e}}$ satisfy the relation

$$
[\mathrm{C}]_{\Omega_{e}}[\mathrm{O}]_{\Omega_{e}}=K_{c o \Omega}
$$

From equations (5.1) and (5.2)

$$
\begin{aligned}
& {[\mathrm{C}]-[\mathrm{C}]_{\Omega_{e}}} \\
& {[\mathrm{C}\rceil-[\mathrm{O}]_{\Omega}}
\end{aligned}=\frac{3}{4} \frac{D_{o}}{D_{c}}=\frac{1}{1}
$$

Substituting equation (5.3) into (5.4), and putting $[\mathrm{O}] \ll[\mathrm{C}] \Lambda$, one has

$$
[\mathrm{O}]_{\Omega e}=\frac{K_{\cos }}{[\mathrm{C}]-[\mathrm{O}] / \Lambda}
$$

Using the limiting value of $[\mathrm{O}]_{\Omega e}$ at $[\mathrm{O}] \rightarrow 0$, equation (5.1) becomes the same equation as the one given by Darken, that is

$$
-\frac{d[\mathrm{C}]}{d t}=\frac{3}{4} D_{o}[\mathrm{O}]-\lfloor\mathrm{O}] c e \frac{S_{g}}{l \Delta l}
$$

The difference between the present equation (5.1) and Darken's equation (5.6) is shown by the following factor:

$$
[\mathrm{O}]-[\mathrm{O}]_{\Omega e} \cong\left([\mathrm{O}]-[\mathrm{C}]_{c e}\right)-\frac{K_{c o}[\mathrm{O}]}{[\mathrm{C}]^{2} \Lambda}
$$

The second term on the right hand side represents the difference of concentration between that in Darken's equation and in the present theory. If $[\mathrm{O}]$ is extremely smaller than $[\mathrm{C}]$, the second term is unnecessary. However, the second term may be effective with a decrease of carbon concentration. In other words, the rate of carbon oxidation $-d[\mathrm{C}] / d t$ becomes slower with advancement of the reaction, since $[\mathrm{O}]-[\mathrm{O}]_{\Omega_{e}}<[\mathrm{C}]-$ $[\mathrm{C}]$ se. This prediction is in agreement with some observations, showing a slow rate in high oxygen region ${ }^{10)}$.

\section{Measurement of Carbon Oxidation in Liquid Iron by Means of Oxygen Blowing}

\section{Experimental Method}

It is interesting to study the rate of carbonoxygen gas reaction in relation to the above mentioned mechanism. This procedure has been popular as a subsidiary technique in the open hearth process.

$$
\underline{\mathrm{C}}+\frac{1}{2} \mathrm{O}_{2}(\mathrm{~g}) \rightarrow \mathrm{CO}(\mathrm{g})
$$

The experimental method for this problem is as follows. An alumina Tammann tube is charged with $\mathrm{Fe}-\mathrm{C}$ alloy containing about $4 \%$ carbon weighing $40 \mathrm{~g}$ and set in a gas tight silica tube. When the alloy has been molten by induction heating, a mixture of nitrogen and oxygen gas, which is adjusted concerning its molar ratio, is induced in the depth of $5 \sim 8 \mathrm{~mm}$ into the liquid alloy through a high alumina blowing pipe (I.D. $6 \mathrm{~mm}$ ). The amount of gas mixture entering into the liquid alloy is checked by a flow meter. CO gas generated due to carbon oxidation is measured by being converted into $\mathrm{CO}_{2}$ through $\mathrm{CuO}$ maintained at $350 \sim$ $400^{\circ} \mathrm{C}$ and by being absorbed in soda lime by means of the ordinary combustion method.

\section{Experimental Results}

The rates of the present reaction measured at various flow rates of the gas mixture whose partial pressure of oxygen is equal to $150 \mathrm{~mm} \mathrm{Hg}$ are represented in Fig. 1, in which carbon dioxide produced by carbon oxidation is plotted against the flow rate of the gas mixture. It is readily seen that the oxygen supplied is completely consumed in the cases of all flow rates. Therefore, it may 


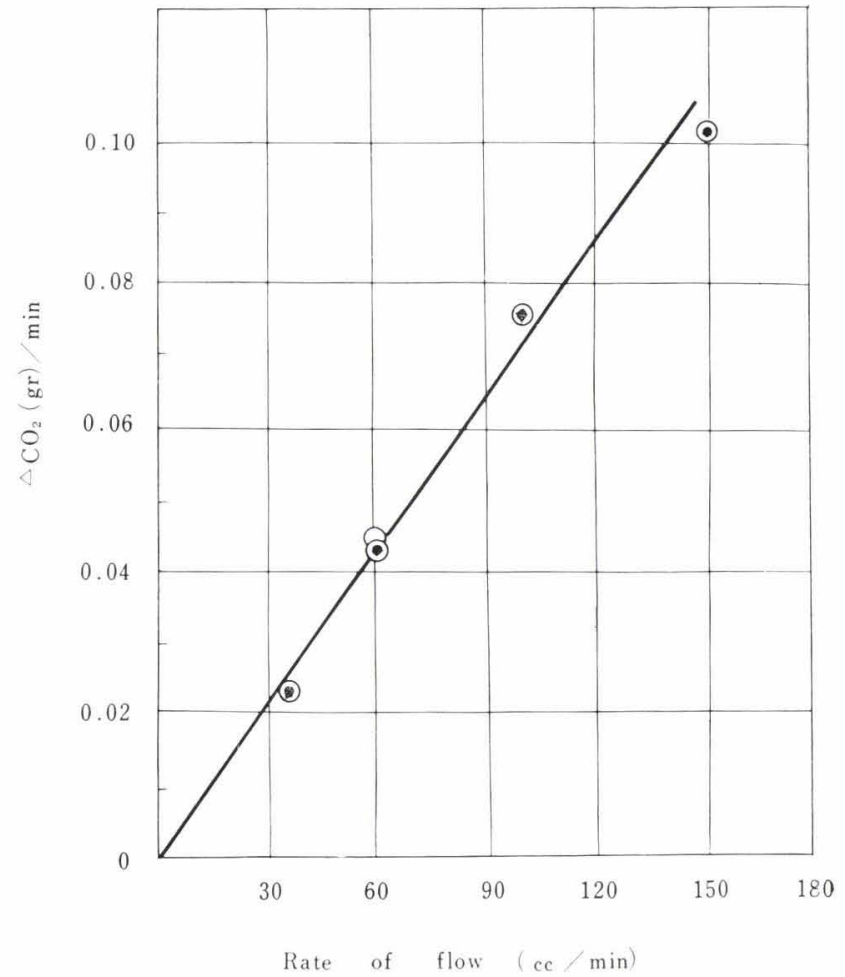

Fig. 1. Yield of carbon dioxide per minute as a function of rate of gas flow at 150 $\mathrm{mm} \mathrm{Hg}$ of $\mathrm{O}_{2}$ pressure and $1550^{\circ} \mathrm{C}$

(Straight line represents the complete consumption of $\mathrm{O}_{2}$.

be emphasized that the rate of the chemical reaction which may occur on the interface between the liquid iron and the gas phase is sufficiently rapid to be settled by a supply of reacting species.

The rates against the variations in oxygen pressure are shown in Table 1 and the degree of decarbonization versus oxygen pressure is represented in Fig. 2. The full line, the best curve through the points over the range of oxygen pressure less than $300 \mathrm{~mm} \mathrm{Hg}$, shows a relation of perfect consumption of oxygen supplied for carbon oxidation, while the dotted line, valid for oxygen pressure higher than $300 \mathrm{~mm} \mathrm{Hg}$, represents the constant rate of carbon oxidation independent of oxygen pressure. This is probably due to the fact that the rate-determining factor exists in a sluggishness of transfer of reactants (i.e. carbon) towards the surface where chemical equilibrium may occur.

\section{Interpretation of the Present Data}

In view of the points indicated in the preceding section, the following assumptions could reasonably be made to explain the experimental results:

i) When the amount of oxygen supply is very small, the rate of the reaction is determined by the
Table 1. Effect of partial pressure of oxygen gas on carbon oxidation

\begin{tabular}{c|c|c|c}
$\begin{array}{c}\text { Partial pressure } \\
\text { of } \mathrm{O}_{2}(\mathrm{mmHg})\end{array}$ & $\begin{array}{c}\text { Flow rate } \\
(\mathrm{cc} / \mathrm{min})\end{array}$ & $\begin{array}{c}\mathrm{CO}_{2} \text { produced } \\
\text { per minute } \\
(\mathrm{gr} / \mathrm{min})\end{array}$ & $\begin{array}{c}\text { Efficiency of carbon } \\
\text { oxidation } \\
(\%)\end{array}$ \\
\hline 25 & 60 & 0.009 & 124 \\
\hline 50 & 60 & 0.018 & 120 \\
\hline 50 & 150 & 0.036 & 98 \\
\hline 75 & 55 & 0.020 & 101 \\
\hline 150 & 60 & 0.045 & 102 \\
\hline 150 & 60 & 0.046 & 104 \\
300 & 60 & 0.088 & 100 \\
300 & 60 & 0.086 & 97 \\
380 & 60 & 0.106 & 97 \\
383 & 60 & 0.087 & 79 \\
450 & 60 & 0.097 & 74 \\
450 & 60 & 0.091 & 69 \\
600 & 60 & 0.106 & 62 \\
600 & 60 & 0.106 & 60 \\
600 & 60 & 0.237 & 48
\end{tabular}

Efficiency of carbon oxidation is defined as twice the moles of carbon monoxide divided by the moles of oxygen supplied.

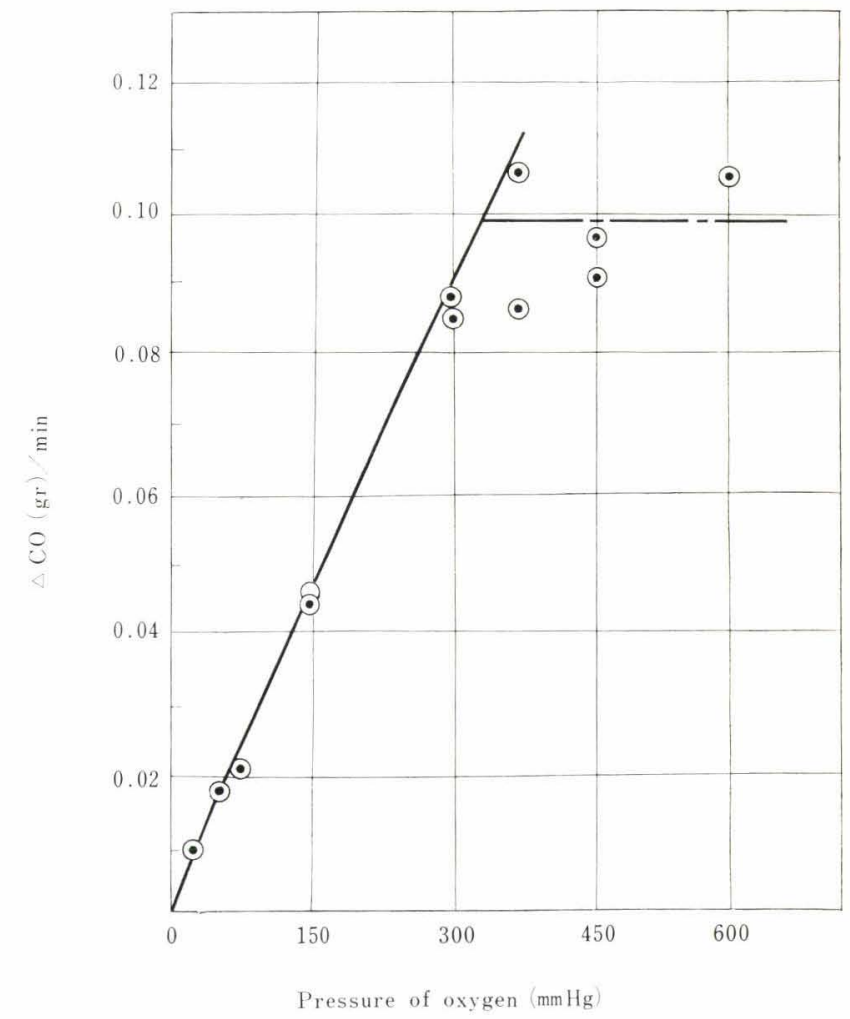

Fig. 2. Yield of $\mathrm{CO}_{2}$ per minute as a function of partial pressure of $\mathrm{O}_{2}$, with fixed flow rate, $60 \mathrm{cc} / \mathrm{min}$, at $1550^{\circ} \mathrm{C}$ 
rate of the oxygen supplied. ( $100 \%$ efficiency).

ii) When the amount of oxygen supply is too large, the rate of the reaction is determined by the flow of carbon through the boundary layer (diffusion film) near the bubbles. (less than $100 \%$ efficiency). For this case we have equation (6.2) from the discussion of the diffusion film theory.

$$
-\frac{d[\mathrm{C}]}{d t}=\frac{D_{c} S_{9 m}}{V} \quad[\mathrm{C}]-[\mathrm{C}] \Omega
$$

where $V$ is the volume of liquid iron, and $S_{g_{m}}$ is the surface area of gas bubbles in contact with liquid iron. Integrating equation (6.2) from time $t_{i}$ to $t$, the amount of carbon atoms removed from liquid iron to a single bubble is evaluated by

$$
\xi=\frac{\rho V}{1200}\left([\mathrm{C}]_{i}-[\mathrm{C}]_{\Omega}\right)\left\{1-\exp \left[\begin{array}{c}
D_{c} S_{g_{m}}\left(t-t_{i}\right) \\
V \Delta l
\end{array}\right]\right\}(6.3)
$$

where $[\mathrm{C}]_{i}$ is the initial concentration of carbon and $\xi$ is expressed in terms of the number of gram atoms of carbon. Considering $D_{c} \cong 1 \times 10^{-4} \mathrm{~cm}^{2} / \mathrm{sec}$, $\Delta l \cong 0.01 \mathrm{~cm}$ and $V \cong 6 \mathrm{cc},(6.3)$ becomes

$$
\xi=\frac{\rho D_{c} S_{g m}}{1200 \Delta l}\left(t-t_{i}\right)\left\{[\mathrm{C}]_{i}-[\mathrm{C}]_{\Omega}\right\}
$$

as long as $S_{g_{m}}\left(t-t_{i}\right) \ll 10^{3} \mathrm{~cm}^{2} / \mathrm{sec}$. For a single gas bubble, emerged from the blowing tube, which reacts with carbon in liquid iron equation (6.4) may be reasonable, because the bubble is very short in life and small in surface area due to its shallow depth and its narrow outlet. The total amount of carbon taken away from liquid iron is

$$
\Xi=\sum_{n} \xi_{n} \cong \begin{gathered}
\rho n \\
1200
\end{gathered} \cdot \frac{D_{c} S_{q m}\left(t-t_{o}\right)}{\Delta l}\left\{\begin{array}{c}
{[\mathrm{C}\rceil_{1 i}+[\mathrm{C}]_{n i}-[\mathrm{C}] \Omega} \\
2
\end{array}\right\}
$$

where $\xi_{n}$ means the amount of carbon for the $n$th bubble and $[\mathrm{C}]_{n i}$ is the initial concentration in the $n$th bubble. $S_{g_{m}}$ and the life of a single bubble $\left(t-t_{o}\right)$ are assumed to be common to every bubble. Furthermore,

$$
\sum_{n}[\mathrm{C}]_{n}=n \begin{gathered}
{[\mathrm{C}]_{1 i}+[\mathrm{C}]_{n i}} \\
2
\end{gathered}
$$

Thus, numerical evaluation of equation (6.5) gives approximately

$$
\Xi \cong 1 \times 10^{-2} S_{q m}\left(t-t_{i}\right)
$$

in units of mole $/ \mathrm{min}$. It is supposed that the order of magnitude of $S_{g_{m}}\left(t-t_{i}\right)$ might be $10^{-1}$ in the present experiment. This value $\left(\Xi \cong 1 \times 10^{-3}\right)$ is in rough agreement with observed values in the region of oxygen pressure higher than $380 \mathrm{~mm} \mathrm{Hg}$, since $3 \times 10^{-3}$ (mole/min) corresponds to yield in Fig. 2. The proposed mechanism from the diffusion film theory may be qualitatively reasonable for an explanation of the present experimental result.

Finally it is to be noted that in present experiment a side reaction such as

$$
\mathrm{Al}_{2} \mathrm{O}_{3}(\mathrm{~S})+3 \mathrm{C} \rightarrow 2 \underline{\mathrm{Al}}+3 \mathrm{CO}(\mathrm{g})
$$

can occur simultaneously with (6.1). The effects of such refractory-metal reactions may correspond to the $120 \%$ efficiency of the reaction (6.1) in Table 1 , according to the preliminary experimental data in this laboratory.

\section{Summary}

The reaction of carbon oxidation in liquid iron,

$$
\mathrm{C}+\mathrm{O} \rightarrow \mathrm{CO}(\mathrm{g})
$$

should be rapid in true terms of homogeneous chemical reaction. This can be confirmed by the fact that

$$
\mathrm{C}+\frac{1}{2} \mathrm{O}_{2}(\mathrm{~g}) \rightarrow \mathrm{CO}(\mathrm{g})
$$

is very rapid in the present experiments, as well as by the theoretical reason of the absolute reaction rate theory. The most reasonable view for the rate-determining step of the above reaction may be obtained from the "diffusion film theory". Then, the thickness of the diffusion layer is briefly discussed in terms of the Raynolds number of liquid iron. The origin and growth of $\mathrm{CO}$ gaseous bubble are explained from the point of view of the "theory of heterogeneous phase transformation" and the "theory of diffusion film". It is to be noted that the effect of viscosity is limited to the narrow region near the boundary interfaces between heterophases but leads to an apparently slow chemical reaction owing to the retarding transport of materials.

\section{REFERENCES}

1) S. Glasstone, K. J. Laidler \& H. Eyring: “ Theory of Rate Process", McGraw-Hill Book, New York, (1941)

2 S. Fornander: Trans. Faraday Soc. "Discussion" No. 4, 1948, pp. 296-307

3 ) L. S. Darken: AIME "Basic Open Hearth Steelmaking" 1951, pp. 621-690

4) L. A. Shvartzman, A. M. Samarin \& M. I. Temkin : J. Phys. Chem. (U. S. S. R.) 21, 1947, p. 1027

$5)$ L. S. Darken \& R. W. Gurry: "Physical Chemistry of Metals" McGraw-Hill Book, 1953

6 ) B. M. Larsen: AIME "Basic Open Hearth Steelmaking" 1951, pp. 867-903 and "A New Look at the Nature of the Open-Hearth Process", AIME (1956)

7) N. A. Parlee, S. R. Seagle, \& R. Schumann, Jr. Trans. Met. Soc. AIME, February 1958, pp. 132-138

8 R. Becker \& W. Döring, Ann. Phys. 24, 1935, p. 732

9 ) W. E. Garner: "Chemistry of the Solid State", Chapter 6, Butterworth Scientific Pub. (1955)

10) T. Fujii: Report of 19th Sectional Committee, Japan, No. $4682(1957)$ 